\title{
Measurement of rise time of averaged muscle action potential in normal subjects and patients with myopathy
}

\author{
A J R SIMONS
}

From the Department of Clinical Neurophysiology, St Antonius Hospital, Utrecht, the Netherlands

SUMMARY As a result of diminution of the number of muscle fibres in patients with myopathy, the rise time of the fast negative phase of the spike potentials in the EMG becomes shorter. By using a small special purpose computer (averager) a mean action potential is obtained from which the rise time of the "spike" is determined. In normal muscles the mean value is 509 microseconds (SD 118). Myopathic muscles show a mean value of 225 microseconds (SD 76). There is a slight overlap and uncertainty between 280 and 370 microseconds. Because of the short time needed to examine the muscle for this parameter many muscles may be examined during one routine EMG.

Measurement of the duration of the motor unit action potential is a well-established EMG method in patients with suspected myopathy. ${ }^{1-3}$

However, during routine electromyography, these measurements are rather difficult and timeconsuming. Not only is it difficult to get welldefined action potentials, because the co-operation of the patient is necessary but the determination of the duration of the action potential is also uncertain because the exact point of the beginning and end of the deflections is often difficult to locate on the oscilloscope or photographic recording.

In a study designed to determine the precision of the method, a number of different experienced electromyographers measured the same photographed recording of action potentials. It was found that very different results were obtained (Myography Group, TNO, Netherlands, 1961, unpublished). However, an individual person may develop an expertise whereby he himself may obtain reproducible and useful results.

The examination may be improved and simplified by using a delay line. ${ }^{4}$ However, the method remains very laborious and is seldom used in routine clinical electromyography.

The rationale of Buchthal's method is founded on the fact that in myopathy isolated muscle fibres degenerate, thereby losing their ability to generate an action potential. Because the motor

Accepted 12 June 1979 unit potential is a summation potential, the amplitude and duration are determined by the number of active fibres/motor unit in which the so-called "temporal dispersion" is an important factor. ${ }^{5}$ Thus the motor unit potential may be shorter in myopathy. Eventually only one action potential of a simple fibre of a degenerating motor unit may remain and the duration of the motor unit potential is very short.

The loss of muscle fibres may also lead to the occurrence of deflections in the summation potential because the remaining fibres of the motor unit give rise to more or less isolated action potentials with a fixed time relation. Thus the picture of polyphasic potentials is produced. These potentials are of very short duration, about 150 microseconds. It should be clear that the loss of single fibre potentials at the beginning and end of a motor unit potential will lead to a shortening of the duration of the latter. Moreover the occurrence of many isolated potentials also gives rise to a shortening of the mean duration of the motor unit potentials.

The most characteristic part of the isolated action potential is the short negative phase, the so-called spike. The duration is about 100 microseconds; the rise time may be as short as 60 microseconds. ${ }^{6-8}$ In the summation potential of the motor unit this phase is much longer, 1-2 milliseconds. Thus, diminution of the number of muscle fibres within the motor unit not only 
shortens the motor unit potential but also the rise time of the negative phase, because, as a result of the smaller number of single fibre potentials the summating and averaging effect becomes less. Thus one may expect that the rise time will be shorter in myopathic muscles.

We therefore looked for a method in which the rise time of the motor unit potential could ke determined easily. The method has to be used in routine EMG examination and should be simple and fast. The results should be available during the examination and be as objective as possible. This calls for a method of automatic analysis.

Because the rise time of a single motor unit potential provides insufficient information, one has to examine several motor unit potentials whose values can be averaged. Potentials which are generated within a distance of $0.5 \mathrm{~mm}$ from the needle point still give values which can be used. Within this radius one may find 10-30 motor units, ${ }^{5}$ and thus it should be possible to detect and use the potentials of many motor units with one insertion of the needle electrode.

\section{Method}

We use an electronic averaging technique to obtain a mean motor unit potential. In this potential the rise time of the spike may be determined as follows.

A concentric needle electrode (Disa No $9013 \mathrm{k} 0031 / 9013 \mathrm{k} 0032$ ) is inserted perpendicularly into the muscle. The muscle should contract, so as to get a mixed EMG pattern. The needle is shifted slightly until a good potential pattern is obtained on the oscilloscope screen (Disa myograph No 14B80; No 1500)

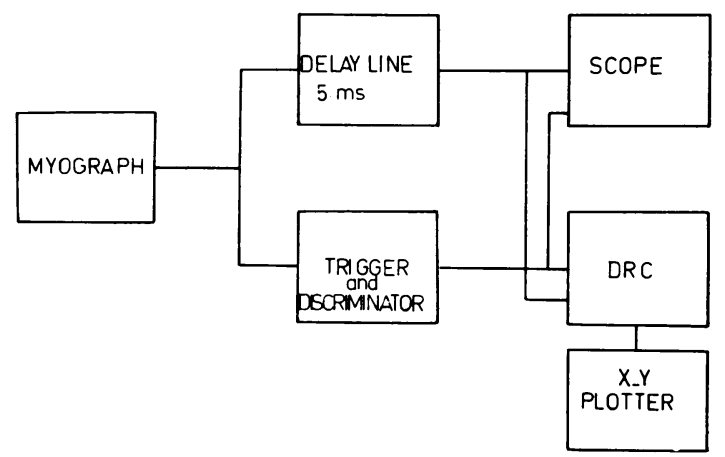

Fig 1 Block diagram of the apparatus for obtaining an averaged motor unit potential.

The output signal of the myograph is fed into (fig 1):

(a) a Schmitt trigger which generates a pulse every time the spike passes a predetermined amplitude level. This level is such that background activity (most of the time of low amplitude from distant action potentials and also slow potentials from artefacts which are attenuated by a suitable high-pass filter) stays below this level. This may be controlled on an oscilloscope screen, (Tektronix No 564B) which shows both the discrimination level and the action potentials (fig 2).

(b) a delay line (Disa No14B80) in which a signal delay of $5 \mathrm{~ms}$ is produced. From this delay line the signal passes to the averaging computer (Nuclear Chicago, DRC 7100), using 400 addresses and a time scale of $15 \mathrm{~ms}$ (cf, LANG $e t a l^{9}$ ).

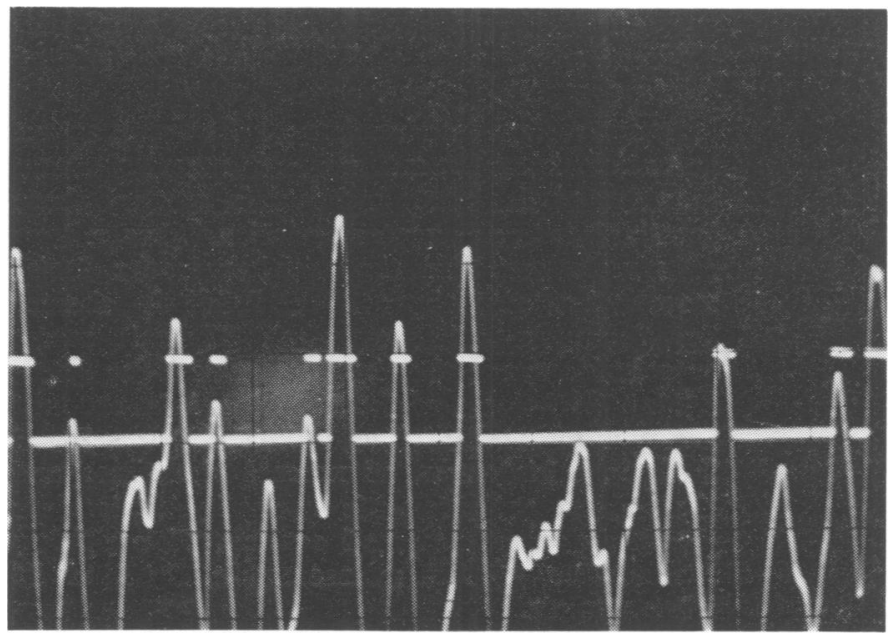

Fig 2 Picture of a number of action potentials and the trigger pulses, generated at the moment the potential passes a predetermined level (time bar $=20 \mathrm{~ms}$ ). 
The above-mentioned pulse triggers the computer. Two hundred potentials arising from about 10-30 motor units ${ }^{5}$ are averaged, to which average another 200 potentials are added, obtained from another part of the same muscle. In general this is sufficient: adding another 200 action potentials from a different part of the muscle does not significantly alter the average. The mean action potential thus obtained in normal muscle resembles in many aspects an isolated action potential but with a duration which is much longer than that of the single muscle fibre potential. The picture of this potential is plotted on an X-Y recorder. The time between successive points of the plot equals 37.5 microseconds. The rise time can then be measured quite easily (fig 3).

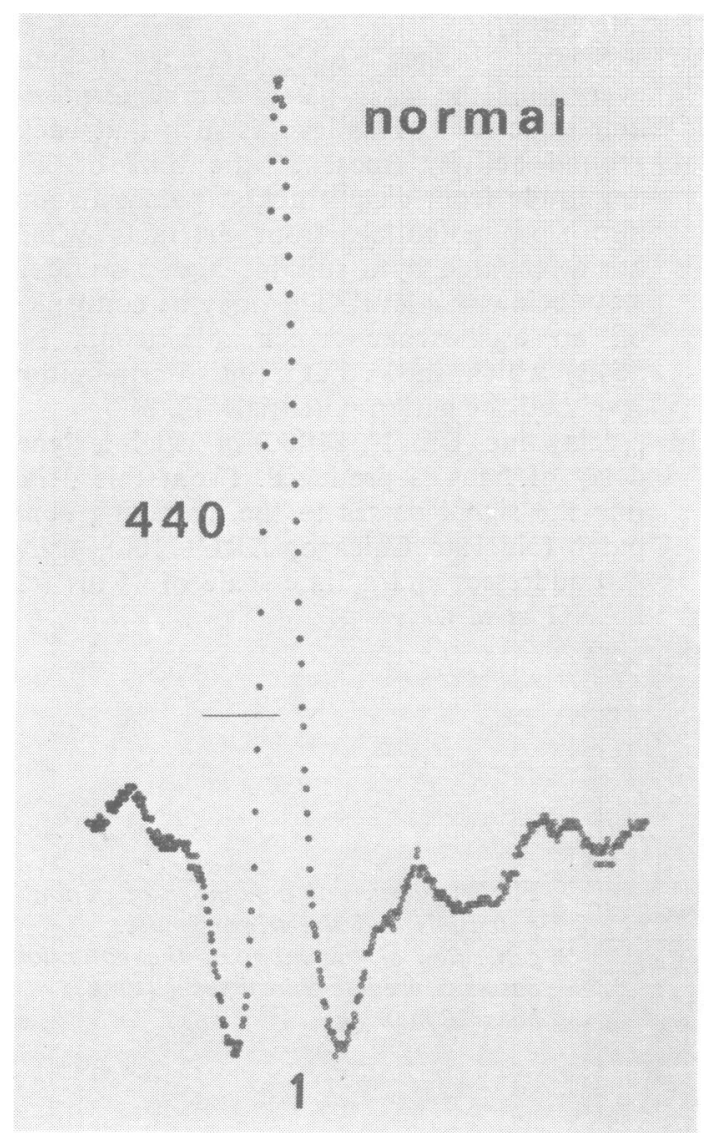

Fig 3 Picture of the average of a number $(n=2 \times 200)$ of normal motor unit potentials (time bar $=15 \mathrm{~ms}$ ).

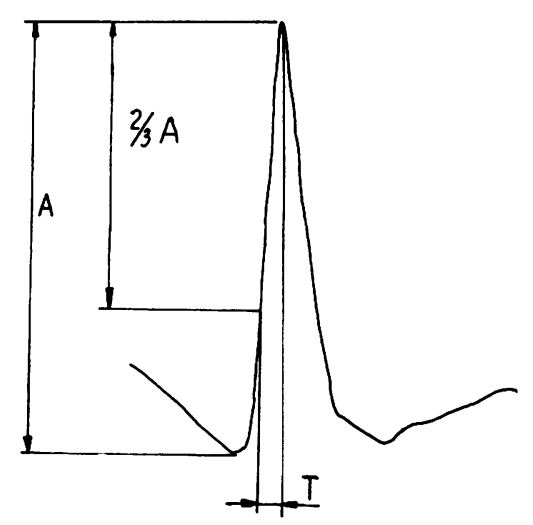

Fig 4 Schematic drawing of the manner of measuring the rise time of the mean motor unit potentials. $A=$ amplitude; $T=$ rise time.

Because the initial deflection of the mean action potential before the spike is poorly defined the time is measured from that part of the spike which is $66 \%$ of the height, taken from the top (fig 4). It has a very constant shape which makes the measurement quite easy and simple.

\section{Results}

As can be seen from fig 5 , the mean action potentials of normal muscles resemble each other closely.

The mean action potentials of myopathic muscles are shown in fig 6 . As a rule there is, besides a considerable shortening of the rise time, a quite different shape, especially of the declining phase of the "spike", as a result of the averaging of polyphasic components of the different motor units which are not time related to the first rising phase.

In measuring the rise time of the mean action potential in different normal muscles, from shoulder girdle, arm, and leg, in general there is no evident and constant relation between type of muscle and rise time (although the facial muscle has rise times of a definitely shorter duration than other muscles). Thus it seems permissible to use the rise time as such for determining pathology, without taking into account the type of the muscle examined. Figures 7 and 8 show a histogram of normal rise times and those from patients with myopathy. It is clear that there is only a small overlap. Figure 7 has a resolution of 100 microseconds, fig 8 of 50 microseconds. 


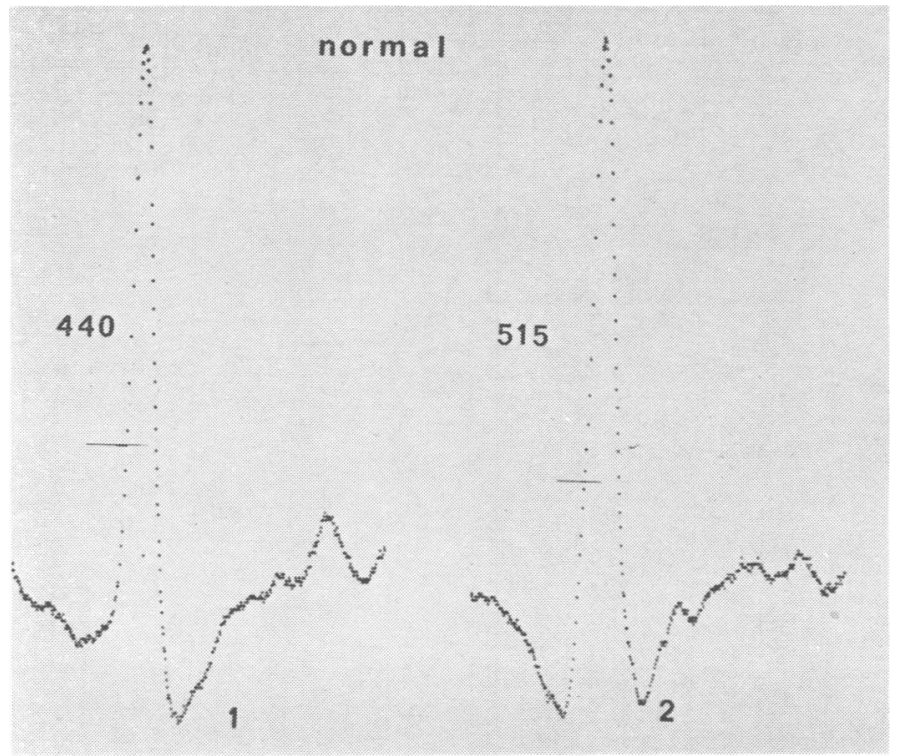

Fig 5 Example of the morphology of two different mean motor unit potentials. The numbers indicate the value of the rise time in microseconds (time bar $=$ $15 \mathrm{~ms}$ ).
The calculated values are:

$\begin{array}{lcrc} & \text { mean } & \text { SD } & \begin{array}{c}95 \% \\ \text { confidence limits }\end{array} \\ \text { Normal subjects }(n-118): & 509 & 118 & 740-280 \\ \text { Myopathies }(n-67): & 225 & 76 & 270-75\end{array}$

Clearly at values between 280 and 370 microseconds it is not possible to determine whether the muscle is normal or myopathic.

There is a clustering of values at $125-150$ microseconds. Here the characteristics of the equipment determine the results. Below 150 microseconds the values are influenced by the highest frequency which may pass the amplifier and delay line.

\section{Discussion}

In electromyography one has to cope with high frequency phenomena of which display and quantification are rather time-consuming procedures and thus most of the time prohibitive in routine clinical EMG. This may be one of the main reasons why measurement of the duration of the muscle action potential ${ }^{1011}$ is not used more commonly.

Another difficulty is to determine which of the recorded action potentials to select for calculation of the mean duration (Myography Group, TNO, 1961, unpublished).

With the method just described, the duration of the motor unit is of no importance, only the rise time of the spike, which may be any spike of a (polyphastic) action potential. Eviery $15 \mathrm{~ms}$ a new trigger pulse may activate the computer, and this trigger pulse may be generated by any spike which reaches the predetermined level. Because the spikes of polyphasic potentials (especially from myopathic muscles) are very short, these spikes determine the value of the average. Nevertheless the polyphasic potential as a whole may be rather long.

A subjective factor in this method is still the choice of the potential series. It is known that the localisation of the needle point in relation to the motor unit may be of importance with respect to the morphology of the motor unit potential. During the examination, after insertion of the needle perpendicular to the direction of the muscle fibres and with contraction until a mixed EMG pattern is obtained, the needle is moved or rotated until the potentials have distinct spike components. In practice this is easy to accomplish but a certain amount of co-operation from the patient is necessary.

Some myopathic muscles show areas with fairly normal motor units and areas with patholcgically altered motor units. If the average is taken from both areas, this will be unreliable. In those cases it may be necessary to determine the average from more than two areas.

A limiting factor is the sensitivity of the instruments used in determining the rise time. The shortest time which can be measured with our computer is 37.5 microseconds. We know that rise times of isolated fibre potentials have values of 60-100 microseconds. ${ }^{6-8}$ The computer thus 

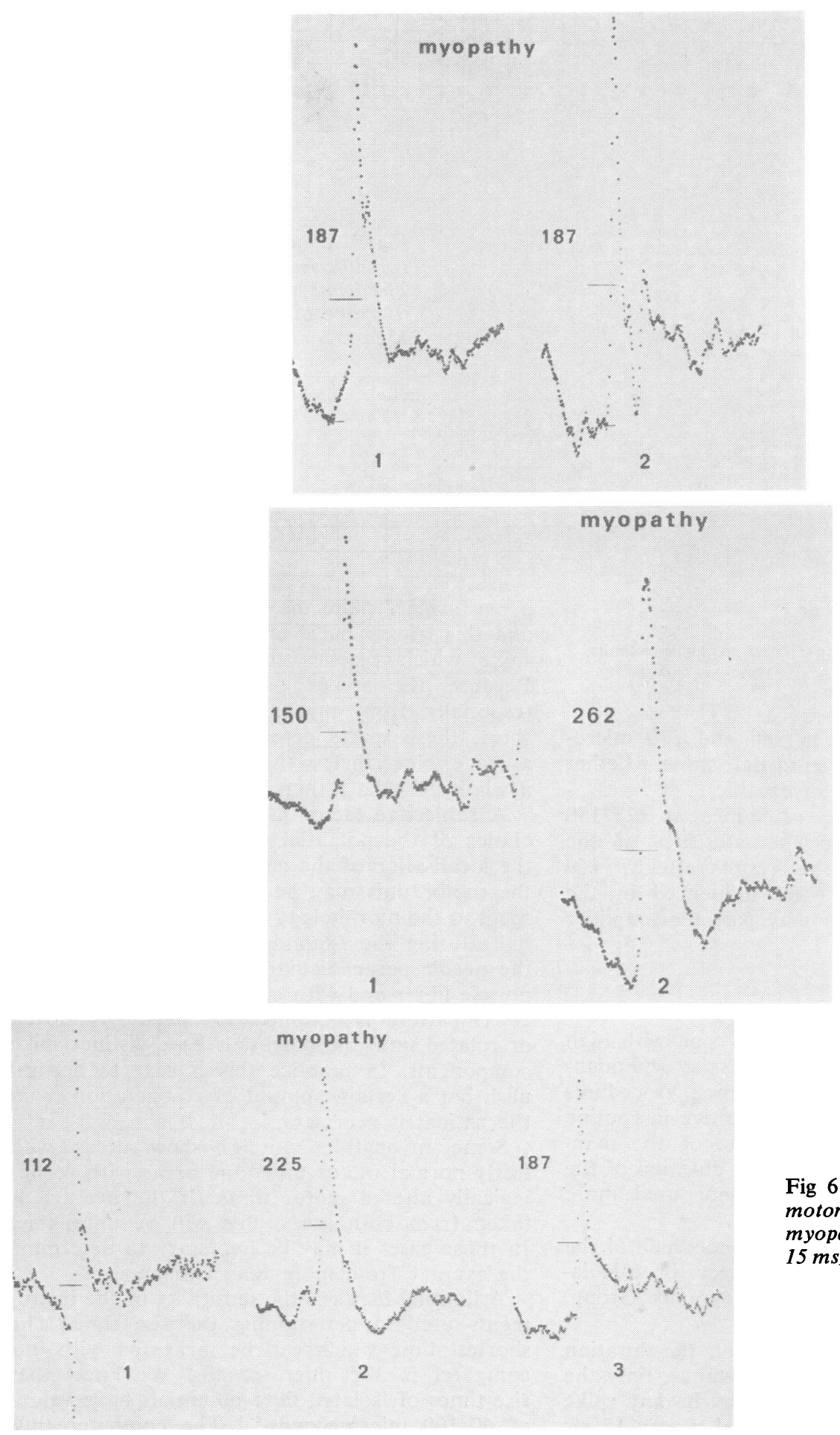

Fig 6 Example of the mean motor unit potentials of myopathic muscles (time bar= $15 \mathrm{~ms}$ ). 


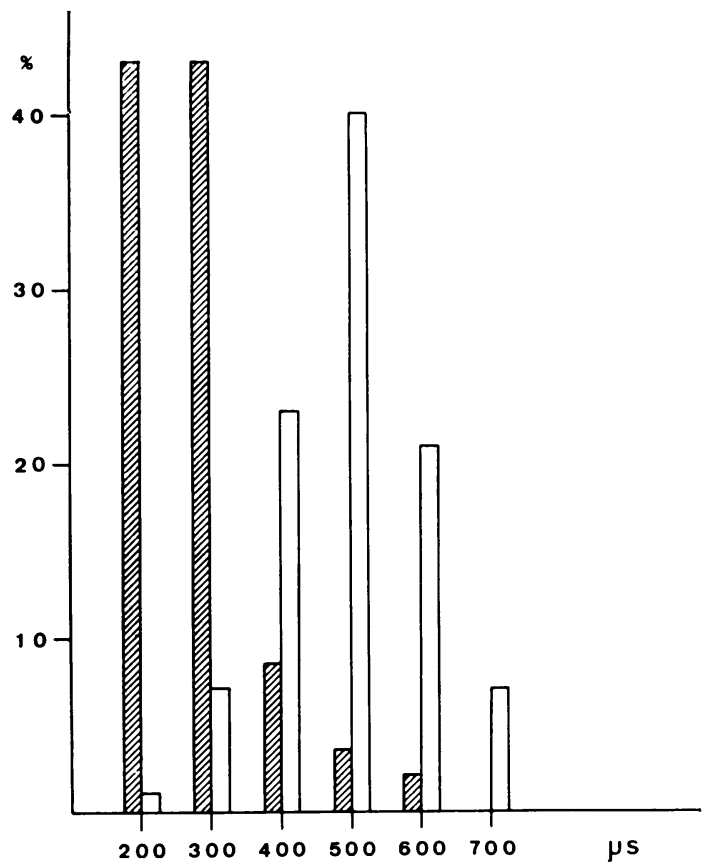

Fig 7 Histogram of spike rise time in normal $\square(n=112)$ and myopathic $\square(n=57)$ muscle.

does not significantly limit the measurements. The delay line, however, limits the measurement of the rise time to 100 microseconds. ${ }^{8}$ The myograph has about the same limitation.

If the upper frequency limit of the apparatus is shifted to a higher value, one would expect to be able to measure shorter rise times, but in

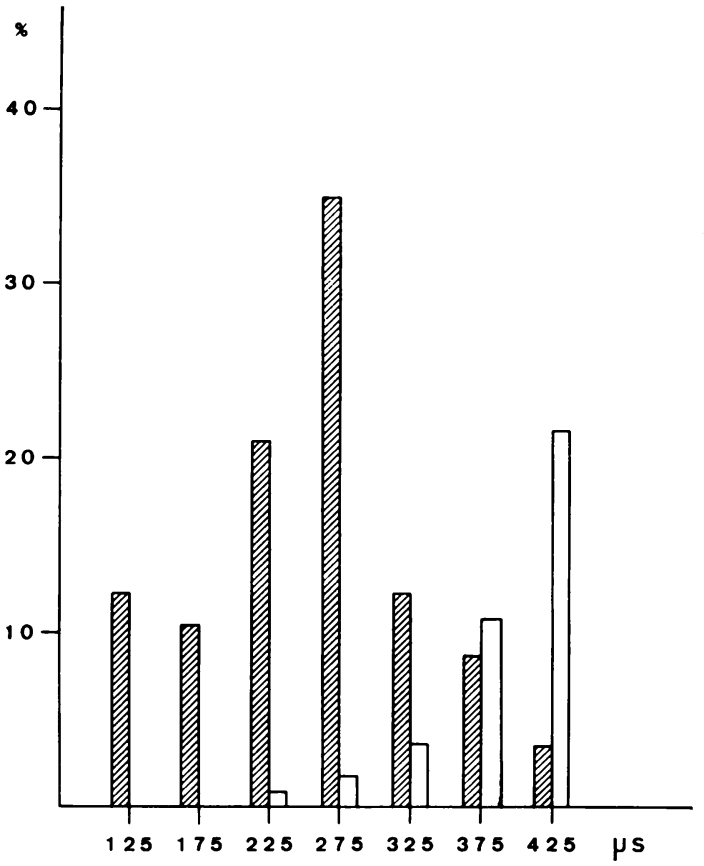

Fig 8 As fig 7 but with greater resolution in the region of shorter rise times.

practice a value of 100 microseconds is so far below the normal value that this will give no more diagnostic information then values between 100 and 250 microseconds.

We measured a number of fibrillation potentials to determine the capability of the system (fig 9). It is clear that this is quite sufficient.

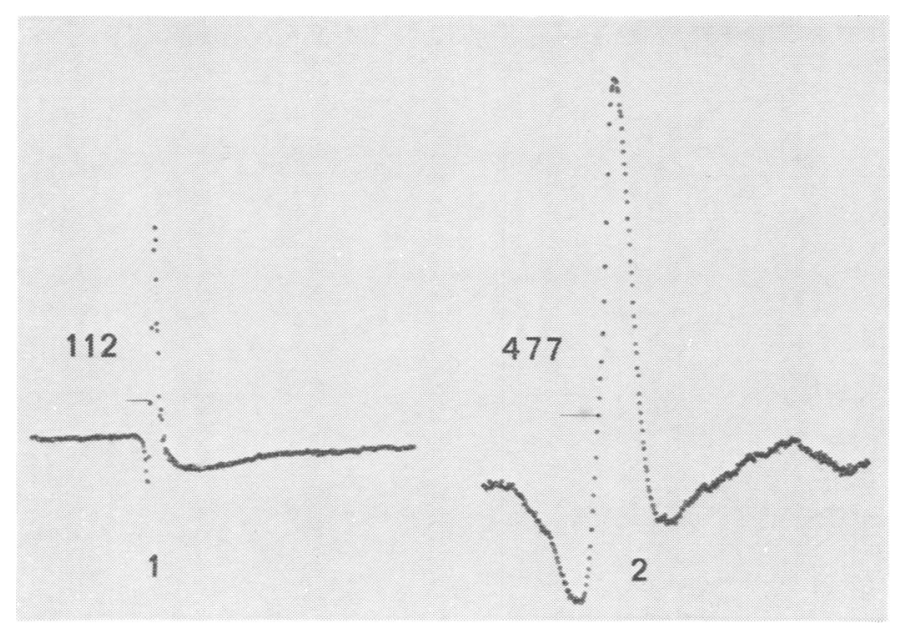

Fig 9 Example of a mean fibrillation potential compared with an average of a normal motor unit potential (time bar $=15 \mathrm{~ms}$ ). 
We have found the method a useful addition to routine diagnostic procedures in myopathy. Because the time needed for one measurement is about five minutes, it is possible to examine many muscles during the routine EMG and to have the results on-line, which augments the diagnostic value of the method.

Technical assistance was provided by $\mathrm{S}$ de Boer and J. Belgraver. J van Doorn helped with the drawings.

\section{References}

1 Buchthal F, Pinelli P. Analysis of action potentials as diagnostic aid in neuro-muscular disorders. Acta Med Scand 1952; Supplement 266: 315-27.

2 Buchthal F, Rosenfalck P. Action potential parameters in different human muscles. Acta Psych Neurol Scand 1955; 30:125-31.

3 Buchthal F. An Introduction to Electromyography. Copenhagen: Scandinavian University Books, Gyldendal, 1957.

4 Nissen-Petersen H, Guld C, Buchthal F. a delayline to record action potentials. Electroencephalogr Clin Neurophysiol 1969; 26:100-6.
5 Buchthal F, Rosenfalck P. Electrophysiological aspects of myopathy with particular reference to progressive muscular dystrophy. In: Bourne GH, Golarz MN, eds. Muscular Dystrophy in Man and Animal. Basel: Karger, 1963: 194-262.

6 Knutsson E, Widen L. Impulses from single nerve fibres recorded in man using microelectrodes. Nature 1967; 213:606-7.

7 Plonsey R, Fleming DG. Bioelectric Phenomena. New York: McGraw Hill, 1969: 1-18.

8 Buchthal F, Dahl K, Rosenfalck P. Rise time of the spike potential in fast and slowly contracting muscle of man. Acta Physiol Scant 1973; 87: 261-9.

9 Lang AH, Nurkkanen P, Vaahtoranta KM. Automatic sampling and averaging of electromyographic unit potentials. Electroencephalogr Clin Neurophysiol 1971; 31:404-6.

10 Buchthal F, Guld G, Rosenfalck P. Action potential parameters in normal human muscle and their dependence on physical variables. Acta Physiol Scand 1954; 32:200-18.

11 Buchthal F, Pinelli P, Rosenfalck P. Action potential parameters in normal human muscle and their physiological determinants. Acta Physiol Scand 1954; 32:219-29. 